\title{
Platelet Distribution Width Level in Patients With Systemic Lupus Erythematosus-Associated Pulmonary Arterial Hypertension and its Diagnostic Value
}

\author{
Yuzhu HE(D), Yikai YU (D), Shaoxian HU(D) \\ Department of Rheumatology and Immunology, Tongji Hospital, Tongji Medical College, \\ Huazhong University of Science and Technology, Wuhan, China
}

\begin{abstract}
Objectives: This study aims to compare the platelet distribution width (PDW) level in patients with systemic lupus erythematosus-associated pulmonary arterial hypertension (SLE-PAH) with that in patients with systemic lupus erythematosus alone (SLE-non-PAH) and to evaluate the clinical value of the PDW level in the early diagnosis of SLE-PAH.

Patients and methods: We analyzed 80 SLE-PAH patients ( 1 males, 79 females; $34.9 \pm 12.3$ years; range, 19 to 77 years) and 154 sex-and age-matched

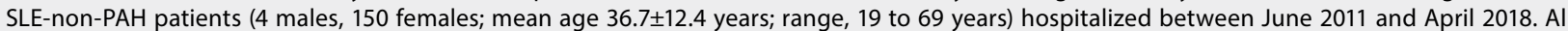
patients underwent transthoracic Doppler echocardiography within three months of inclusion in the study. Age, sex, disease course, currently prescribed medications, clinical manifestations, and past history were collected. Pulmonary artery systolic pressure, ejection fraction, white blood cell count, red blood cell count, hemoglobin, platelet count, PDW, mean platelet volume, erythrocyte sedimentation rate, complement 3 (C3), and C4 levels were also obtained.

Results: The PDW level was higher in the SLE-PAH group than that in the SLE-non-PAH group $(p=0.023)$. SLE patients were allocated into high systemic lupus erythematosus disease activity index (SLEDAI) group (SLEDAI score, $\geq 10)(n=121)$ or low SLEDAl group (SLEDAI score, $<10)$ ( $n=113)$. The PDW level was significantly higher in the high SLEDAI group than that in the low SLEDAI group ( $p=0.030)$. The receiver operating characteristic curve was used to evaluate the clinical value of the PDW level in diagnosing PAH in SLE patients. The PDW level was valuable for diagnosing PAH in SLE patients [area under the curve $(A \cup C)=0.591, p=0.023$ ]. The optimal critical value of the PDW level was $14.55 \mathrm{fL}$. Under these conditions, the sensitivity, specificity, and Youden index were $57 \%, 63 \%$ and 0.20 , respectively. For newly diagnosed patients, the PDW level had good diagnostic accuracy, with an AUC of $0.626(\mathrm{p}=0.037)$. The optimal critical value of the PDW level was $14.65 \mathrm{fL}$. Under these conditions, the sensitivity, specificity, and Youden index were $66 \%, 67 \%$ and 0.33 , respectively.
\end{abstract}

Conclusion: The PDW level is a good predictor of SLE-PAH, and this parameter is applicable to various clinical settings.

Keywords: Platelet distribution width, predictor, pulmonary arterial hypertension, systemic lupus erythematosus.

Systemic lupus erythematosus (SLE) is a systemic autoimmune disease characterized by the presence of circulating autoimmune antibodies that damage multiple organs within the cardiovascular system, respiratory system, urinary system, and hematological system. ${ }^{1}$ Pulmonary arterial hypertension is comprised of a group of diseases characterized by the progressive increase of pulmonary resistance. The prevalence of SLE with pulmonary arterial hypertension (SLE-PAH) is significantly lower than that of the common clinical manifestations of SLE, such as lupus nephritis, arthritis, and hematological system involvement. Regardless, PAH is the third leading cause of death in SLE patients, and it is an independent risk factor for death in this

Received: September 01, 2019 Accepted: November 10, 2019 Published online: February 07, 2020

Correspondence: Shaoxian Hu, MD. Department of Rheumatology and Immunology, Tongji Hospital, Tongji Medical College, Huazhong University of Science and Technology, 430030 Wuhan, China. Tel: 8615271870760 e-mail: shaoxianhu123@163.com

He Y, Yu Y, Hu S. Platelet Distribution Width Level in Patients With Systemic Lupus Erythematosus-Associated Pulmonary Arterial Hypertension and its Diagnostic Value. Arch Rheumatol 2020;35(3):394-400 
population. The five-year survival rate of patients with SLE-PAH is much lower than that of patients with SLE alone..$^{2,3}$ Although PAH is not a common complication of SLE, the diagnosis and treatment of SLE-PAH should receive more attention due to its poor prognosis.

The early clinical manifestations of SLE$\mathrm{PAH}$ are not always specific for the disease. Furthermore, there are few studies describing the biomarkers of SLE-PAH, and a lack of reliable biomarkers further complicates the diagnosis of the disease. Timely treatment can lengthen the survival time of SLE-PAH patients, so it is important to identify new biomarkers for the early diagnosis of SLE-PAH. ${ }^{4}$

Platelet distribution width (PDW) level is an indicator of the heterogeneity in platelet (PLT) size. An elevated PDW level indicates increased PLT activation. 5,6 Some studies have showed that PDW and mean PLT volume (MPV) are valuable biomarkers of immunological diseases. ${ }^{7-9}$ A previous study has reported an elevated PDW level in SLE patients, as well as a positive correlation between the PDW level and disease severity, suggesting that the PDW level is a predictor of SLE activity. ${ }^{10}$ PLT volume indexes are widely used as predictors of cardiovascular diseases. ${ }^{11-14}$ Recently, Zheng et al. ${ }^{15}$ found that the PDW level was elevated in patients with idiopathic pulmonary arterial hypertension (IPAH). However, to our knowledge, there is no study on the clinical value of the PDW level for diagnosing SLE-PAH. Therefore, in this study, we aimed to compare the PDW level in patients with SLE-PAH with that in patients with systemic lupus erythematosus alone (SLE-non-PAH) and to evaluate the clinical value of the PDW level in the early diagnosis of SLE-PAH.

\section{PATIENTS AND METHODS}

A total of 80 SLE-PAH patients (1 males, 79 females; $34.9 \pm 12.3$ years; range, 19 to 77 years) as well as 154 sex- and age-matched SLE-non$\mathrm{PAH}$ patients (4 males, 150 females; mean age $36.7 \pm 12.4$ years; range, 19 to 69 years) hospitalized at Tongji Hospital, Tongji Medical College, Huazhong University of Science and Technology between June 2011 and April 2018 were retrospectively analyzed. All patients met the diagnostic criteria for SLE, as revised by the American Society of Rheumatology in 1997 or the International Clinical Collaboration Group for SLE in 2012. Patients aged <16 years or those with a history of recent blood transfusion, recent pregnancy, IPAH, coronary heart disease, congenital heart disease, heart valve disease, chronic obstructive pulmonary disease, hematological system disease, cancer, serious infection, or other connective tissue diseases were excluded. All patients underwent transthoracic Doppler echocardiography within three months of inclusion in the study. The pulmonary artery systolic pressure (PASP) (mmHg) was estimated by tricuspid regurgitation. The PASP in the SLE-PAH group was $\geq 40 \mathrm{mmHg} .{ }^{16}$ The study protocol was approved by the Tongji Hospital, Tongji Medical College, Huazhong University of Science and Technology Ethics Committee. A written informed consent was obtained from each patient. The study was conducted in accordance with the principles of the Declaration of Helsinki.

The age, sex, disease course, currently prescribed medications, clinical manifestations, and past history were collected. The PASP, ejection fraction $(\mathrm{EF})$, white blood cell count, red blood cell count (RBC), hemoglobin ( $\mathrm{Hb})$, PLT count, PDW, MPV, erythrocyte sedimentation rate (ESR), complement $3(\mathrm{C} 3)$, and $\mathrm{C} 4$ levels were also obtained. The severity of SLE was evaluated by the systemic lupus erythematosus disease activity index (SLEDAI).

\section{Statistical analysis}

When two samples met the conditions of normal distribution and homogeneous variance, the t-test was used and results were described by mean \pm standard deviation; otherwise, the Mann-Whitney test was used and results were described by median $\left(25^{\text {th }}\right.$ percentile, $75^{\text {th }}$ percentile). When two samples met the condition of normal distribution, Pearson's correlation analysis was used; otherwise, Spearman's correlation analysis was used.

Counting data were presented as percentages. The four-grid table was used to determine whether there was a significant difference in the sample rate between the two groups.

The receiver operating characteristic ( $\mathrm{ROC}$ ) curve was used to evaluate the clinical value of 


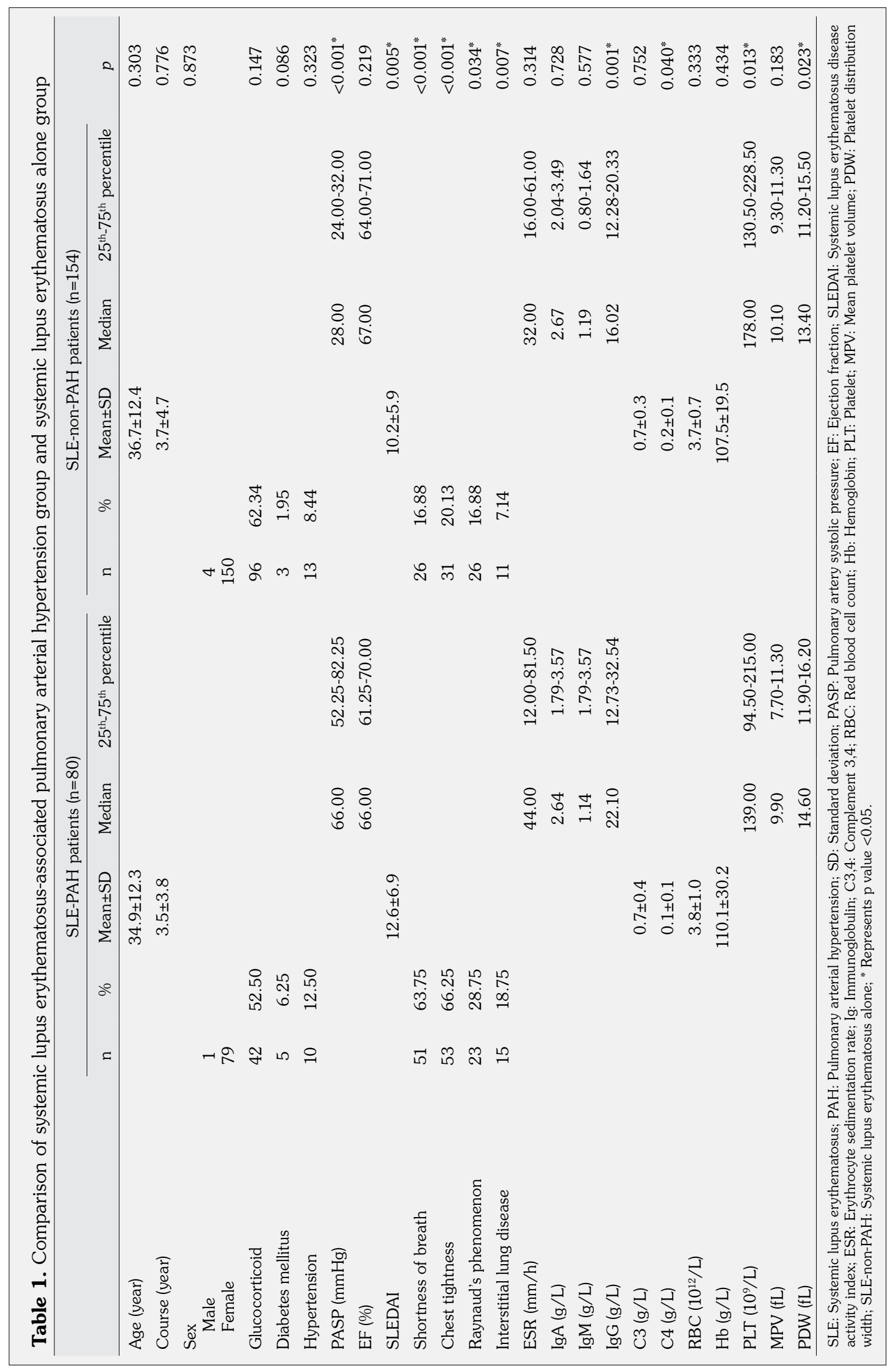


the PDW level for diagnosing SLE-PAH. The $\mathrm{p}$ value and area under the curve (AUC) were calculated. The sensitivity, specificity, and Youden index corresponding to the best critical value were also calculated. A $p$ value $<0.05$ was considered statistically significant. The IBM SPSS version 22.0 (IBM Corp., Armonk, NY, USA) software was used for statistical analysis.

\section{RESULTS}

No significant differences in age, disease course, sex ratio, glucocorticoid use ratio, and proportions of diabetes mellitus and hypertension were observed between the SLE-PAH and SLE-non-PAH groups (all $p$ values $>0.05$ ). The PASP of the SLE-PAH group was higher than that of the SLE-non-PAH group $(p<0.001)$, whereas there was no difference in the EF between the groups $(p=0.219)$. The SLEDAI score of the SLE-PAH group was significantly higher than that of the SLE-nonPAH group $(p=0.005)$. The incidence rates of shortness of breath, chest tightness, Raynaud's phenomenon, and interstitial lung disease in the SLE-PAH group were higher than those in the SLE-non-PAH group (all $p$ values $<0.05$ ). Thirtyeight patients were allocated into the newly diagnosed SLE-PAH group, whereas 58 patients were allocated into the newly diagnosed SLEnon-PAH group. Newly diagnosed patients had similar clinical characteristics (Tables 1 and 2).

The PDW level as well as the immunoglobulin (Ig) G concentration were higher in the SLE-PAH group than those in the SLE-non-PAH group (all $p$ values $<0.05$ ). The serum C4 and PLT values in the SLE-PAH group were lower than those in the SLE-non-PAH group (all $p$ values $<0.05)$. No statistical differences in the ESR and the levels of $\operatorname{IgA}, \operatorname{IgM}, \mathrm{C} 3, \mathrm{RBC}, \mathrm{Hb}$, and $\mathrm{MCV}$ were observed between the SLE-PAH and SLE-non-PAH groups (all $p$ values $>0.05$ ). For newly diagnosed SLE patients, the PDW level in the SLE-PAH group was also higher than that in the SLE-non-PAH group $(p=0.049)$. SLE patients were allocated into high SLEDAI group (SLEDAI score, $\geq 10)(\mathrm{n}=121)$ or low SLEDAI group (SLEDAI score, <10) $(\mathrm{n}=113)$. The PDW level in the high SLEDAI group was $14.80(11.30,16.00) \mathrm{fL}$, which was significantly higher than that in the low SLEDAI group of $(p=0.030)$. Newly diagnosed SLE patients were allocated into high SLEDAI group $(n=59)$ or low SLEDAI group $(n=36)$. PDW level in the high SLEDAI group was also higher (0.015) (Tables 1, 2 and 3).

Table 2. Comparison of initially diagnosed systemic lupus erythematosus-associated pulmonary arterial hypertension patients and systemic lupus erythematosus alone patients

\begin{tabular}{|c|c|c|c|c|c|c|c|c|c|c|c|}
\hline & \multicolumn{5}{|c|}{ SLE-PAH patients $(\mathrm{n}=38)$} & \multicolumn{5}{|c|}{ SLE-non-PAH patients $(\mathrm{n}=58)$} & \multirow[b]{2}{*}{$p$} \\
\hline & $\mathrm{n}$ & $\%$ & Mean \pm SD & Median & $\begin{array}{l}25^{\text {th }}-75^{\text {th }} \\
\text { percentile }\end{array}$ & $\mathrm{n}$ & $\%$ & Mean \pm SD & Median & $\begin{array}{l}25^{\text {th }}-75^{\text {th }} \\
\text { percentile }\end{array}$ & \\
\hline Age (year) & & & $33.6 \pm 13.8$ & & & & & $35.3 \pm 12.1$ & & & 0.522 \\
\hline Course (year) & & & & 1.00 & $0.08-2.50$ & & & & 0.25 & $0.08-1.00$ & 0.108 \\
\hline Sex & & & & & & & & & & & 1.000 \\
\hline $\begin{array}{l}\text { Male } \\
\text { Female }\end{array}$ & $\begin{array}{c}1 \\
37\end{array}$ & & & & & $\begin{array}{c}2 \\
56\end{array}$ & & & & & \\
\hline Diabetes mellitus & $1 / 38$ & 2.63 & & & & $1 / 58$ & 1.72 & & & & 1.000 \\
\hline Hypertension & $4 / 38$ & 10.53 & & & & $5 / 58$ & 8.62 & & & & 0.737 \\
\hline PASP (mmHg) & & & & 58.50 & $52.00-76.25$ & & & & 27.00 & $24.00-32.00$ & $<0.001^{*}$ \\
\hline $\mathrm{EF}(\%)$ & & & & 65.50 & $61.00-70.00$ & & & & 67.00 & $63.00-71.00$ & 0.167 \\
\hline SLEDAI & & & $12.5 \pm 6.1$ & & & & & $9.9 \pm 5.5$ & & & $0.002^{*}$ \\
\hline $\operatorname{PLT}\left(10^{9} / \mathrm{L}\right)$ & & & & 136.00 & $83.50-255.75$ & & & & 175 & $131.5-207.5$ & 0.203 \\
\hline MPV (fL) & & & & 9.15 & 7.05-11.05 & & & & 10.3 & $9.3-11.40$ & $0.037^{*}$ \\
\hline PDW (fL) & & & & 15.15 & $11.85-16.28$ & & & & 13.9 & $11.35-15.05$ & $0.049^{*}$ \\
\hline
\end{tabular}

SLE: Systemic lupus erythematosus; PAH: Pulmonary arterial hypertension; SD: Standard deviation; PASP: Pulmonary artery systolic pressure; EF: Ejection fraction; SLEDAI: Systemic lupus erythematosus disease activity index; PLT: Platelet; MPV: Mean platelet volume; PDW: Platelet distribution width; SLE-nonPAH: Systemic lupus erythematosus alone; ${ }^{*}$ Represents $p$ value $<0.05$. 
Table 3. Comparison of platelet distribution width level in high systemic lupus erythematosus disease activity index group with that in low systemic lupus erythematosus disease activity index group

\begin{tabular}{|c|c|c|c|c|c|c|c|c|c|c|}
\hline \multirow{3}{*}{$\begin{array}{l}\text { Grouping } \\
\text { PDW (fL) }\end{array}$} & \multicolumn{4}{|c|}{ All SLE patients $(\mathrm{n}=234)$} & \multirow[b]{2}{*}{$p$} & \multicolumn{4}{|c|}{ Newly diagnosed patients $(\mathrm{n}=96)$} & \multirow[b]{2}{*}{$p$} \\
\hline & \multicolumn{2}{|c|}{ SLEDAI $\geq 10(\mathrm{n}=121)$} & \multicolumn{2}{|c|}{ SLEDAI $<10(\mathrm{n}=113)$} & & \multicolumn{2}{|c|}{ SLEDAI $\geq 10 \quad(\mathrm{n}=56)$} & \multicolumn{2}{|c|}{ SLEDAI $<10(\mathrm{n}=36)$} & \\
\hline & 14.80 & $11.30-16.00$ & 12.90 & $11.30-15.20$ & 0.030 & 14.8 & $12.30-15.90$ & 12.20 & $11.10-15.00$ & 0.015 \\
\hline
\end{tabular}

Table 4. Evaluation of diagnostic value for systemic lupus erythematosus-associated pulmonary arterial hypertension of PDW and sUA by receiver operating characteristic curve

\begin{tabular}{|c|c|c|c|c|c|c|c|}
\hline Test variable & AUC & $95 \% \mathrm{CI}$ & Best critical value (fL) & Sensitivity (\%) & Specificity (\%) & Youden index & $p$ \\
\hline PDW & 0.591 & $0.510-0.672$ & 14.55 & 57 & 63 & 0.20 & $0.023^{*}$ \\
\hline
\end{tabular}

Table 5. Evaluation of diagnostic value for systemic lupus erythematosus-associated pulmonary arterial hypertension of PDW and sUA in newly diagnosed patients

\begin{tabular}{|c|c|c|c|c|c|c|c|}
\hline Test variable & AUC & $95 \% \mathrm{CI}$ & Best critical value (fL) & Sensitivity (\%) & Specificity (\%) & Youden index & $p$ \\
\hline PDW & 0.626 & $0.502-0.751$ & 14.65 & 66 & 67 & 0.33 & $0.037^{*}$ \\
\hline
\end{tabular}

The ROC curve was used to evaluate the clinical value of the PDW level for diagnosing $\mathrm{PAH}$ in SLE patients. The PDW level had certain diagnostic value, with an AUC of 0.591 $(p=0.023)$. The optimal critical value of the PDW

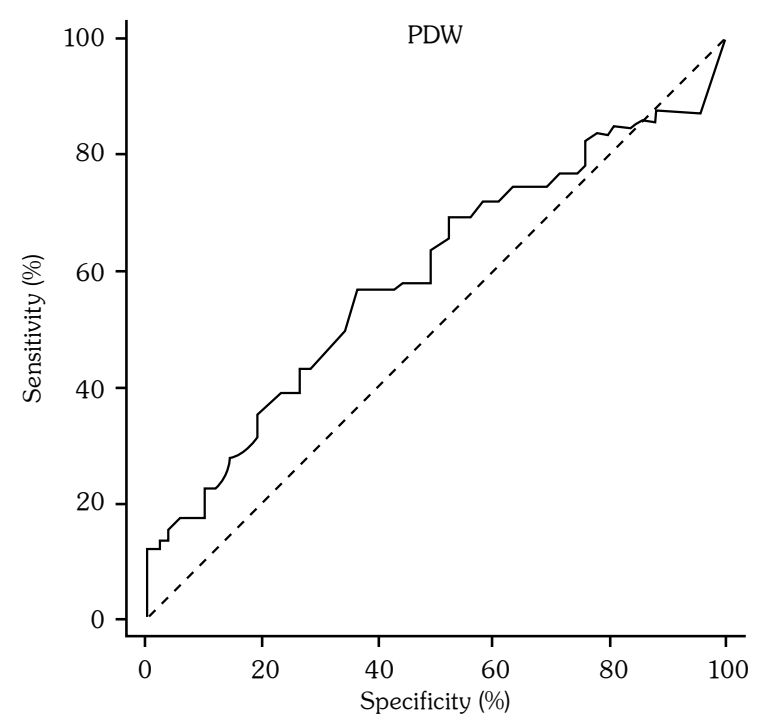

Figure 1. Value of platelet distribution width for diagnosing pulmonary arterial hypertension in systemic lupus erythematosus patients.

PDW: Platelet distribution width. level was $14.55 \mathrm{fL}$. Under these conditions, the sensitivity, specificity, and Youden index were 57\%, 63\% and 0.20, respectively. For newly diagnosed patients, the PDW level had good diagnostic accuracy, with an AUC of

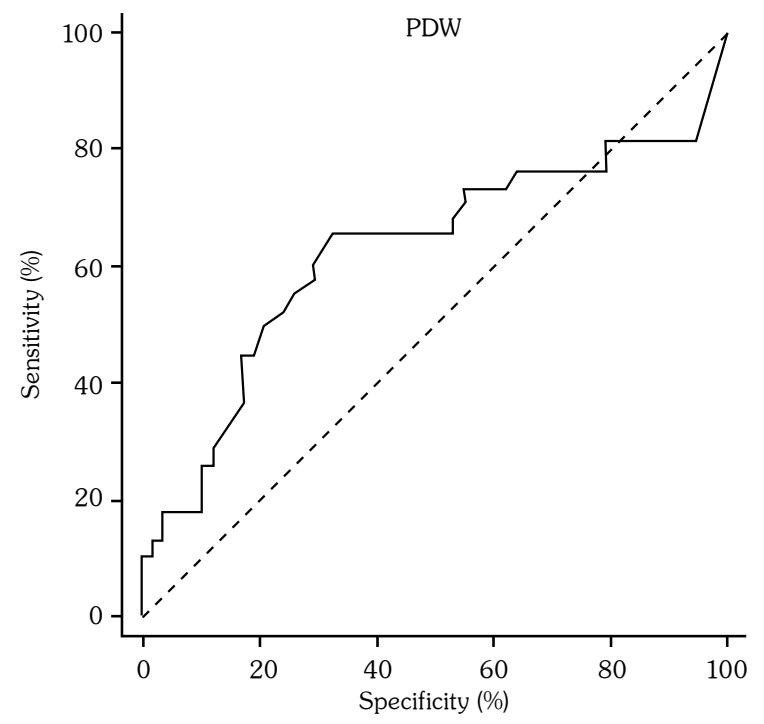

Figure 2. Value of platelet distribution width for diagnosing pulmonary arterial hypertension in newly diagnosed systemic lupus erythematosus patients. PDW: Platelet distribution width. 
0.626 ( $p=0.037)$. The optimal critical value of the PDW level was $14.65 \mathrm{fL}$. Under these conditions, the sensitivity, specificity, and Youden index were 66\%, 67\% and 0.33 , respectively (Tables 4 and 5 and Figures 1 and 2).

\section{DISCUSSION}

The clinical symptoms of SLE-PAH are not specific to the disease, as similar symptoms have been reported for other rheumatoid, cardiovascular, respiratory, and renal disorders. In addition, there are few reliable biomarkers for the diagnosis of the disease. The aim of this study was to identify a new biomarker for the early diagnosis of SLE-PAH.

In this study, no significant differences in the age, disease course, sex ratio, glucocorticoid use ratio, and proportions of diabetes mellitus and hypertension between the SLE-PAH and SLE-non-PAH groups were noted.

The PDW level, which reflects the size of PLTs, is a predictor of PLT activation. ${ }^{6}$ PLT activation, which involves inflammatory cytokines and complements, has been observed in patients with SLE. ${ }^{17,18}$ A previous study has reported an elevated PDW level in SLE patients compared to healthy controls, as well as a positive correlation between the PDW level and disease severity. ${ }^{10}$ Therefore, the PDW level may be a reliable marker for the clinical assessment of SLE. Another study has indicated that the PDW level was higher in patients with IPAH than that in healthy controls; ${ }^{15}$ thus, the PDW level may also reflect the severity of IPAH.

We found that the PDW level was higher in the SLE-PAH group than that in the SLE-non-PAH group. As discussed above, an elevated PDW level, which was observed in patients with SLE or IPAH, indicates increased PLT activation. In this study, the SLEDAI score, which reflects the severity of SLE, was higher in the SLE-PAH group than that in the SLE-non-PAH group. The PDW level was higher in the high SLEDAI group than that in the low SLEDAI group, suggesting that the PDW level can reflect the severity of SLE at least to some degree. SLE patients with higher disease severity may have greater systemic inflammation, which can lead to PLT activation and an elevated
PDW level. Moreover, increased PLT activation can cause thrombosis, ${ }^{19-20}$ which may contribute to vascular changes in patients with pulmonary arterial hypertension. Furthermore, we observed that the PDW level had certain clinical value in the diagnosis of SLE-PAH. For newly diagnosed SLE patients, the PDW level also had good diagnostic accuracy.

Our study had several limitations. Firstly, the PASP was estimated by tricuspid regurgitation. Although a previous study has defined a PASP of $40 \mathrm{mmHg}$ as good cut-off value for the diagnosis of PAH, heart catheterization is the gold standard for PASP measurement. Secondly, the size of the study sample was not large enough, and further studies are needed to validate our findings.

In conclusion, the prognosis of SLE-PAH is poor. Furthermore, many SLE-PAH patients present with clinical symptoms that are not specific to the disease, which contributes to incorrect and missed diagnoses and to the lack of timely treatment. Recently, SLE-PAH research has gained momentum. In this study, we showed that the PDW level has certain clinical value in the diagnosis of SLE-PAH, and this parameter is applicable to various clinical settings. However, further studies are needed to better reflect its value in the early diagnosis of SLE-PAH.

\section{Declaration of conflicting interests}

The authors declared no conflicts of interest with respect to the authorship and/or publication of this article.

\section{Funding}

The authors received no financial support for the research and/or authorship of this article.

\section{REFERENCES}

1. Agmon-Levin N, Mosca M, Petri M, Shoenfeld Y. Systemic lupus erythematosus one disease or many? Autoimmun Rev 2012;11:593-5.

2. Fei Y, Shi X, Gan F, Li X, Zhang W, Li M, et al. Death causes and pathogens analysis of systemic lupus erythematosus during the past 26 years. Clin Rheumatol 2014;33:57-63.

3. Min HK, Lee JH, Jung SM, Lee J, Kang KY, Kwok $\mathrm{SK}$, et al. Pulmonary hypertension in systemic lupus erythematosus: an independent predictor of patient 
survival. Korean J Intern Med 2015;30:232-41.

4. Galiè N, Rubin Lj, Hoeper M, Jansa P, Al-Hiti H, Meyer $G$, et al. Treatment of patients with mildly symptomatic pulmonary arterial hypertension with bosentan (EARLY study): a double-blind, randomised controlled trial. Lancet 2008;371:2093-100.

5. Osselaer JC, Jamart J, Scheiff JM. Platelet distribution width for differential diagnosis of thrombocytosis. Clin Chem 1997;43:1072-6.

6. Vinholt PJ, Hvas AM, Nybo M. An overview of platelet indices and methods for evaluating platelet function in thrombocytopenic patients. Eur $\mathrm{J}$ Haematol 2014;92:367-76.

7. Korkmaz S. Mean platelet volume and platelet distribution width levels in patients with mild psoriasis vulgaris with metabolic syndrome. Postepy Dermatol Alergol 2018;35:367-71.

8. Kılıç S, Reşorlu H, Issik S, Oymak S, Akbal A, Hız MM, et al. Association between mean platelet volume and disease severity in patients with psoriasis and psoriatic arthritis. Postepy Dermatol Alergol 2017;34:126-30.

9. Soydinc S, Turkbeyler IH, Pehlivan Y, Soylu G, Goktepe MF, Bilici M, et al. Mean platelet volume seems to be a valuable marker in patients with systemic sclerosis. Inflammation 2014;37:100-6.

10. Chen SY, Du J, Lu XN, Xu JH. Platelet distribution width as a novel indicator of disease activity in systemic lupus erythematosus. J Res Med Sci 2018;23:48.

11. Sansanayudh N, Anothaisintawee T, Muntham D, McEvoy M, Attia J, Thakkinstian A. Mean platelet volume and coronary artery disease: a systematic review and meta-analysis. Int $\mathrm{J}$ Cardiol 2014;175:433-40.

12. Khode V, Sindhur J, Kanbur D, Ruikar K, Nallulwar $\mathrm{S}$. Mean platelet volume and other platelet volume indices in patients with stable coronary artery disease and acute myocardial infarction: A case control study. J Cardiovasc Dis Res 2012;3:272-5.

13. Moharamzadeh P, Rahmani F, Foroughifar S, Shahsavarinia K. Reliability of Platelet Indices for Diagnosing Pulmonary Embolism; a Brief Report. Adv J Emerg Med 2019;3:e27.

14. Ozyurtlu F, Yavuz V, Cetin N, Acet H, Ayhan E, Isik $\mathrm{T}$. The association between coronary slow flow and platelet distribution width among patients with stable angina pectoris. Postepy Kardiol Interwencyjnej 2014;10:161-5.

15. Zheng YG, Yang T, Xiong CM, He JG, Liu ZH, Gu $\mathrm{Q}$, et al. Platelet distribution width and mean platelet volume in idiopathic pulmonary arterial hypertension. Heart Lung Circ 2015;24:566-72.

16. Ruiz-Irastorza G, Garmendia M, Villar I, Egurbide MV, Aguirre C. Pulmonary hypertension in systemic lupus erythematosus: prevalence, predictors and diagnostic strategy. Autoimmun Rev 2013;12:410-5.

17. Boilard E, Blanco P, Nigrovic PA. Platelets: active players in the pathogenesis of arthritis and SLE. Nat Rev Rheumatol 2012;8:534-42.

18. Habets KL, Huizinga TW, Toes RE. Platelets and autoimmunity. Eur J Clin Invest 2013;43:746-57.

19. Kamisli O, Kamisli S, Kablan Y, Gonullu S, Ozcan C. The prognostic value of an increased mean platelet volume and platelet distribution width in the early phase of cerebral venous sinus thrombosis. Clin Appl Thromb Hemost 2013;19:29-32.

20. Sevuk U, Bahadir MV, Altindag R, Baysal E, Yaylak $\mathrm{B}$, Ay $\mathrm{N}$, et al. Value of serial platelet indices measurements for the prediction of pulmonary embolism in patients with deep venous thrombosis. Ther Clin Risk Manag 2015;11:1243-9. 